\title{
P wave duration in paroxysmal and persistent atrial fibrillation
}

\author{
Malte Unkell ${ }^{1, A-F}$, Maxim Marinov ${ }^{1, A-F}$, Peter Stephan Wolff ${ }^{1, A-F}$, \\ Jadwiga Radziejewska ${ }^{2, B, F}$, Jakub Szymon Mercik ${ }^{3, A-F}$, Jacek Gajek ${ }^{4, A-F}$ \\ 1 Students' Scientific Association, Department of Emergency Medical Service, Wroclaw Medical University, Poland \\ ${ }^{2}$ Klodzko County Hospital, Poland \\ ${ }^{3}$ Department of Emergency Medicine, Wroclaw Medical University, Poland \\ ${ }^{4}$ Department of Emergency Medical Service, Wroclaw Medical University, Poland \\ A - research concept and design; $\mathrm{B}$ - collection and/or assembly of data; $\mathrm{C}$ - data analysis and interpretation; \\ $D$ - writing the article; $E$ - critical revision of the article; $F$ - final approval of the article
}

Address for correspondence

\section{Jakub Mercik}

E-mail: jakub.mercik@wp.pl

\section{Funding sources}

None declared

Conflict of interest

None declared

Received on June 21, 2020

Reviewed on July 8, 2020

Accepted on September 20, 2020
Cite as

Unkell M, Marinov M, Wolff PS, Radziejewska J, Mercik JS,

Gajek J.P wave duration in paroxysmal and persistent atrial fibrillation. Adv Clin Exp Med. 2020;29(11):1347-1354. doi:10.17219/acem/127680

DOI

10.17219/acem/127680

\section{Copyright}

Copyright by Author(s)

This is an article distributed under the terms of the

Creative Commons Attribution 3.0 Unported (CC BY 3.0)

(https://creativecommons.org/licenses/by/3.0/)

\section{Abstract}

Background. Functional and structural changes in the atrial muscle constitute a substrate for atrial fibrillation (AF). The pathological changes in the left atrium decrease the conduction velocity and result in prolongation of the $P$ wave duration.

Objectives. To assess the duration of the P wave in patients with AF in different clinical presentations of arrhythmia.

Material and methods. The study group consisted of 119 patients diagnosed with AF: 57 women and 62 men, aged $65.3 \pm 9.4$ years. There were 65 patients with paroxysmal AF and 54 with persistent AF. In this group, electrical cardioversion was performed. The $P$ wave duration was measured using an electrophysiological system in all leads at a paper speed of $200 \mathrm{~mm} / \mathrm{s}$.

Results. The patients did not differ in terms of age, gender or comorbidities. The patients with persistent AF had longer $P$ wave duration (159.9 $\pm 22.3 \mathrm{~ms}$ compared to $144.6 \pm 17.2 \mathrm{~ms} ; p<0.001$ ) and higher glucose concentration ( $119.4 \pm 33.4 \mathrm{mg} / \mathrm{dL}$ compared to $108.0 \pm 24.6 \mathrm{mg} / \mathrm{dL} ; p=0.015)$. These results were not influenced by the anti-arrhythmic treatment.

Conclusions. Persistent AF shows a longer P wave duration than the paroxysmal AF, independent of age, gender and anti-arrhythmic medication. The prolongation of the $P$ wave related to persistent arrhythmia should force physicians to restore the sinus rhythm earlier in order to more successfully maintain it in the long term.

Key words: P wave duration, atrial fibrillation, diabetes mellitus, chronic kidney disease 


\section{Introduction}

Atrial fibrillation (AF) is an arrhythmic consequence of multiple pathological processes leading to functional and structural changes in the atrial muscle. ${ }^{1,2}$ Among the major pathologies leading to this arrhythmia, hypertension, coronary artery disease (CAD), and subsequent heart failure (HF) play the main roles. Despite treating both atria as a substrate of AF, it is clinically assumed that this arrhythmia occurs mainly due to left atrial pathologies, primarily due to the higher workload it needs to cope with as a consequence of the higher left ventricular resistance (LVR). These diseases cause changes in the structure of the atrial muscle through death and apoptosis of cardiomyocytes, contributing to stromal fibrosis. This affects the generation of arrhythmia foci, local potential fragmentation and possible re-entry loops. However, the main consequence visible in echocardiography is the left atrial enlargement.

Furthermore, AF is caused by other arrhythmias, such as multiple atrial extrasystole, atrial focal tachycardia or atrial flutter. ${ }^{3-5}$ Whether they are constant or paroxysmal, they lead to electrophysiological changes in the action potential - usually a shortening of the refractory period, the local intensity of which may differ. This is manifested by the heterogeneity of the repolarization process. Repolarization disorders lead to functional conduction disturbances, which - superimposed on the structural changes and conduction slowing associated with cardiomyocyte depletion - intensify the re-entry phenomenon and promote the maintenance and persistence of arrhythmias. These pathologies have an impact on the electrocardiographic (ECG) picture of atrial muscle depolarization, depicted by the $\mathrm{P}$ wave of the electrocardiogram. With the duration and progression of functional and structural changes, the duration of the $\mathrm{P}$ wave is prolonged, making it a risk factor for AF. ${ }^{6}$

An interesting and clinically important issue is the positive relationship between the AF paroxysm and the tendency of the arrhythmia to persist, which was reflected in the phrase "AF begets AF" coined by Wijffels et al. ${ }^{7}$ Rapid atrial arrhythmias affect the functional changes in the process of atrial muscular repolarization and, above all, induce heterogeneity of refraction duration by the formation of local blocks and slow conduction zones. ${ }^{8}$ In addition, AF episodes lead to left atrial enlargement, most likely due to an increase in filling pressure, but also due to blood retention and functional mitral regurgitation. All of these processes over time lead to the paroxysmal AF becoming persistent, until finally the decision is made to leave the arrhythmia in a permanent form. ${ }^{8,9}$

All of these issues indicate the need for complex systemic treatment and prevention of AF paroxysms. An important aspect is to reduce the duration of individual episodes using pharmacological or electrical conversion to a sinus rhythm. Prolonged arrhythmia paroxysms lead to a deepening of functional and anatomical changes, so it is likely that patients with persistent AF after sinus rhythm restoration have a longer $P$ wave duration than patients with the paroxysmal form of the arrhythmia.

The aim of the study was to assess $P$ wave duration in patients with AF in different clinical presentations of the arrhythmia.

\section{Material and methods}

The study group consisted of 119 patients diagnosed with AF. The group consisted of 57 women and 62 men, aged $65.3 \pm 9.4$ years. The essential comorbidities were reported. There were 65 patients with paroxysmal AF (AF group) who had a sinus rhythm during examination, and 54 patients with persistent AF. In this group, electrical cardioversion was performed to restore a sinus rhythm (CV group). Any antiarrhythmic medication, including $\beta$-blockers, propafenone and amiodarone (combination therapy as well), was also recorded. As the exact duration of the arrhythmia episodes was not possible to recollect, we only included those patients with persistent AF lasting 2-24 weeks.

The P wave duration was measured using a LabSystem ${ }^{\mathrm{TM}}$ Pro EP Recording System (Boston Scientific, Boston, USA), where the ECG tracings allowed the sinus P waves to be assessed. The P wave duration was measured precisely in all leads at a paper speed of $200 \mathrm{~mm} / \mathrm{s}$ and an enhancement of 64-128. To avoid any influence of accidental inaccuracies, all measurements were repeated 5 times and the mean value was taken as the result.

In patients with the persistent form of AF, the direct current cardioversion was performed as a standard clinical procedure under general anesthesia using $1 \mathrm{mg} / \mathrm{kg}$ of propofol and $50 \mu \mathrm{g}$ of fentanyl, administered intravenously. A single shock of $300 \mathrm{~J}$ was successful in all patients.

The study protocol was approved by the local Bioethical Committee at Wroclaw Medical University, Poland.

\section{Statistical analysis}

The statistical analysis was performed using STATISTICA v. 13.3 (StatSoft, Inc., Tulsa, USA). P-values less than 0.05 were considered statistically significant.

For quantitative variables, basic descriptive statistics were calculated ( $\mathrm{M}$ - average, $\mathrm{SD}$ - standard deviation, $\mathrm{Me}-\mathrm{me}$ dian, Q1 - lower quartile, Q3 - upper quartile, Min - minimum value, and Max - maximum value) and the compliance of their distributions with a theoretical normal distribution was checked using the Shapiro-Wilk W test. Comparisons were performed with Student's t-test or the Mann-Whitney U test for independent groups, or Kruskal-Wallis analysis of variance (ANOVA) for multiple comparisons. Each categorical variable is presented as numbers and percentages. The comparisons were performed with the $X^{2}$ test. 
The correlations between the parameters were checked using Spearman's rank correlation coefficient according to the statistical properties of the data.

The receiver operating characteristic (ROC) was used to assess the ability of $\mathrm{P}$ wave duration to classify disease status. Based on the results of examination and ROC analysis, a cutoff threshold for P wave duration was calculated for the AF and CV groups.

\section{Results}

The demographic and clinical characteristics of the study participants are presented in Table 1.

The $\mathrm{P}$ wave duration did differ significantly between the 2 groups, lasting longer in patients with the persistent form of arrhythmia. The same applied for fasting glucose concentration. The details are presented in Fig. 1. The $\mathrm{P}$ wave duration was significantly longer in patients with chronic kidney disease (CKD). The results are presented in Fig. 2. Moreover, there was a weak but statistically significant correlation between the mean P wave duration and creatinine concentration. The results are depicted in Fig. 3.

The upper and lower limits of 95\% confidence interval (95\% CI) are greater than 0 , i.e., the correlation is positive and statistically significant $(\mathrm{p}<0.05)$. The increase in creatinine concentration was accompanied by an increase in $\mathrm{P}$ wave duration.

However, no statistically significant relationship was observed between the dosage of propafenone and the duration of the P wave. Spearman's rank correlation cohort rho does not differ significantly from 0 (the upper and lower limits of the $95 \%$ CI differ in sign, i.e., they contain 0; $\mathrm{p}>0.05)$. This conclusion applies to both the entire sample of patients and to the AF and CV groups separately.

There were no correlations between $P$ wave duration and propafenone treatment (dosage) among all patients, nor in AF and CV groups. The results are depicted in Fig. 4. The basic statistics of $\mathrm{P}$ wave duration in the groups
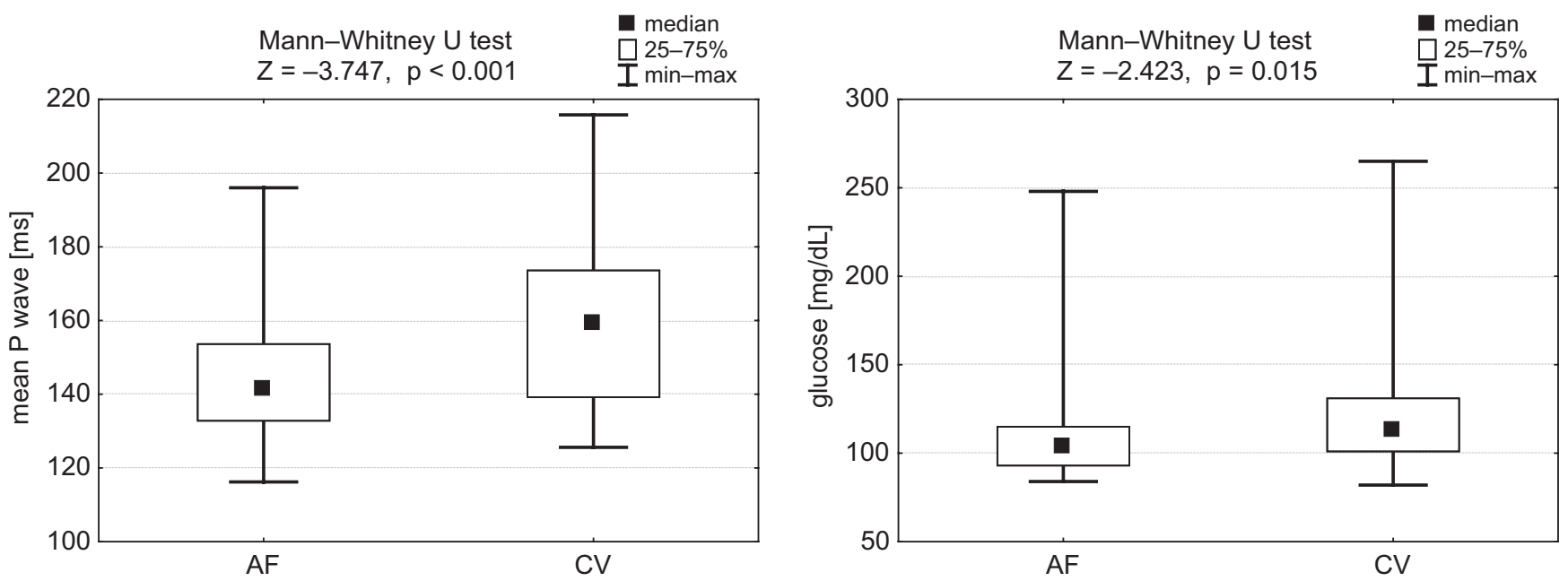

Fig. 1. Glucose level and average $\mathrm{P}$ wave duration in groups of patients according to the type of AF and the results of significance tests

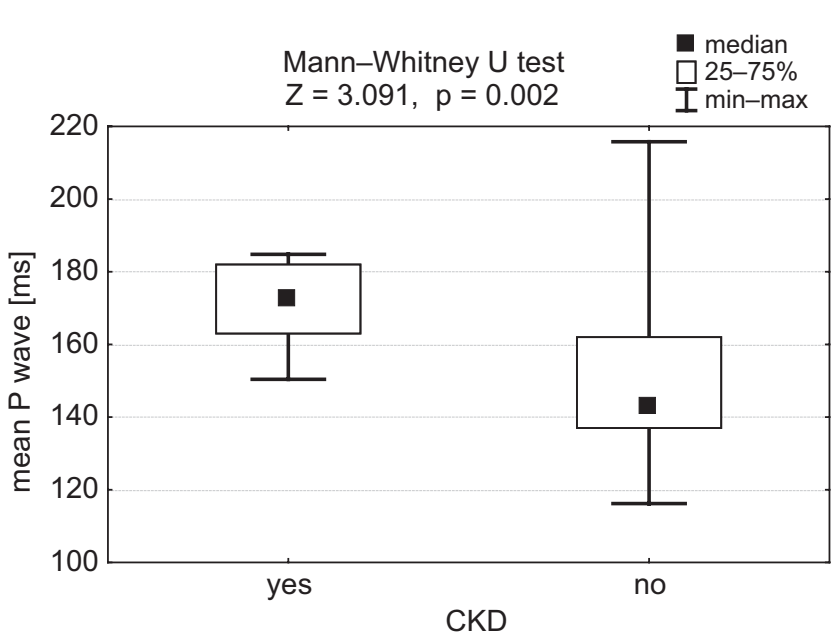

Fig. 2. The average $P$ wave duration in groups of patients according to the presence of CKD and the results of significance tests

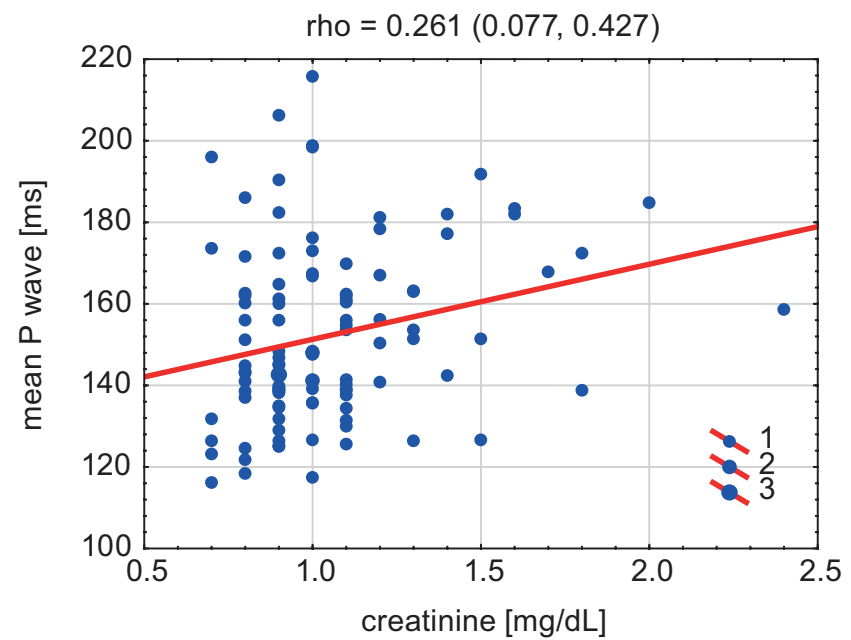

Fig. 3. Correlation diagram of average $P$ wave duration with creatinine level and Spearman's rank correlation coefficient and its $95 \% \mathrm{Cl}$ 
Table 1. Clinical characteristics of the total population and the comparisons of 2 groups of studied patients

\begin{tabular}{|c|c|c|c|c|}
\hline \multirow{2}{*}{ Parameter } & Total & $\mathrm{AF}$ & $\mathrm{CV}$ & \multirow{2}{*}{$p$-value } \\
\hline & $n=119(100 \%)$ & $n=65(54.6 \%)$ & $\mathrm{n}=54(45.4 \%)$ & \\
\hline Mean age [years] & $65.3 \pm 9.4$ & $65.0 \pm 8.9$ & $65.6 \pm 10.1$ & 0.712 \\
\hline Male/female & $62 / 57$ & $34 / 31$ & $28 / 26$ & 0.893 \\
\hline \multicolumn{5}{|c|}{ Comorbidities: } \\
\hline HT & $89(74.8 \%)$ & $48(73.8 \%)$ & $41(75.9 \%)$ & 0.962 \\
\hline DM & $25(21.0 \%)$ & $12(18.5 \%)$ & $13(24.1 \%)$ & 0.601 \\
\hline CKD & $9(7.6 \%)$ & $6(9.2 \%)$ & $3(5.6 \%)$ & 0.509 \\
\hline IHD & $21(17.6 \%)$ & $12(18.5 \%)$ & $9(16.7 \%)$ & 0.989 \\
\hline $\mathrm{HF}$ & $11(9.2 \%)$ & $3(4.6 \%)$ & $8(14.8 \%)$ & 0.065 \\
\hline \multicolumn{5}{|c|}{ Laboratory: } \\
\hline $\mathrm{K}+[\mathrm{mmol} / \mathrm{L}]$ & $4.45 \pm 0.44$ & $4.42 \pm 0.43$ & $4.49 \pm 0.45$ & 0.446 \\
\hline Glucose $[\mathrm{mg} / \mathrm{dL}]$ & $113.3 \pm 29.4$ & $108.0 \pm 24.6$ & $119.4 \pm 33.4$ & 0.015 \\
\hline Creatinine $[\mathrm{mg} / \mathrm{dL}]$ & $1.05 \pm 0.28$ & $1.01 \pm 0.22$ & $1.09 \pm 0.34$ & 0.340 \\
\hline \multicolumn{5}{|c|}{ Medicines: } \\
\hline Propafenone & $43(36.1 \%)$ & 19 (29.2\%) & $24(44.4 \%)$ & 0.126 \\
\hline Amiodarone & $17(14.3 \%)$ & $9(13.8 \%)$ & $8(14.8 \%)$ & 0.910 \\
\hline Bisoprolol & $22(18.5 \%)$ & $12(18.5 \%)$ & $10(10.5 \%)$ & 0.819 \\
\hline Metoprolol & $86(72.3 \%)$ & $44(67.7 \%)$ & $42(77.8 \%)$ & 0.309 \\
\hline Mean P wave duration [ms] & $151.5 \pm 21.0$ & $144.6 \pm 17.2$ & $159.9 \pm 22.3$ & $<0.001$ \\
\hline
\end{tabular}

HT - arterial hypertension; DM - diabetes mellitus; CKD - chronic kidney disease; IHD - ischemic heart disease; HF - heart failure.

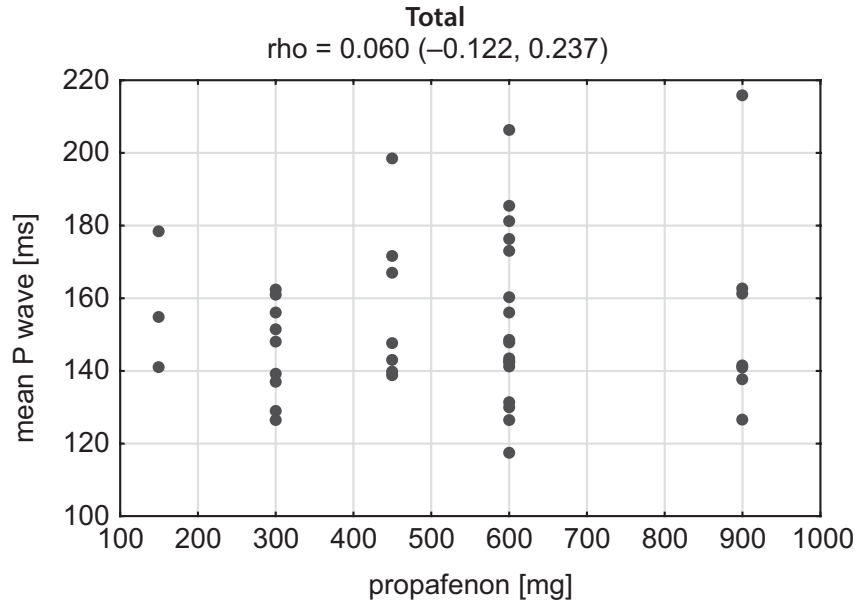

$\mathrm{AF}$
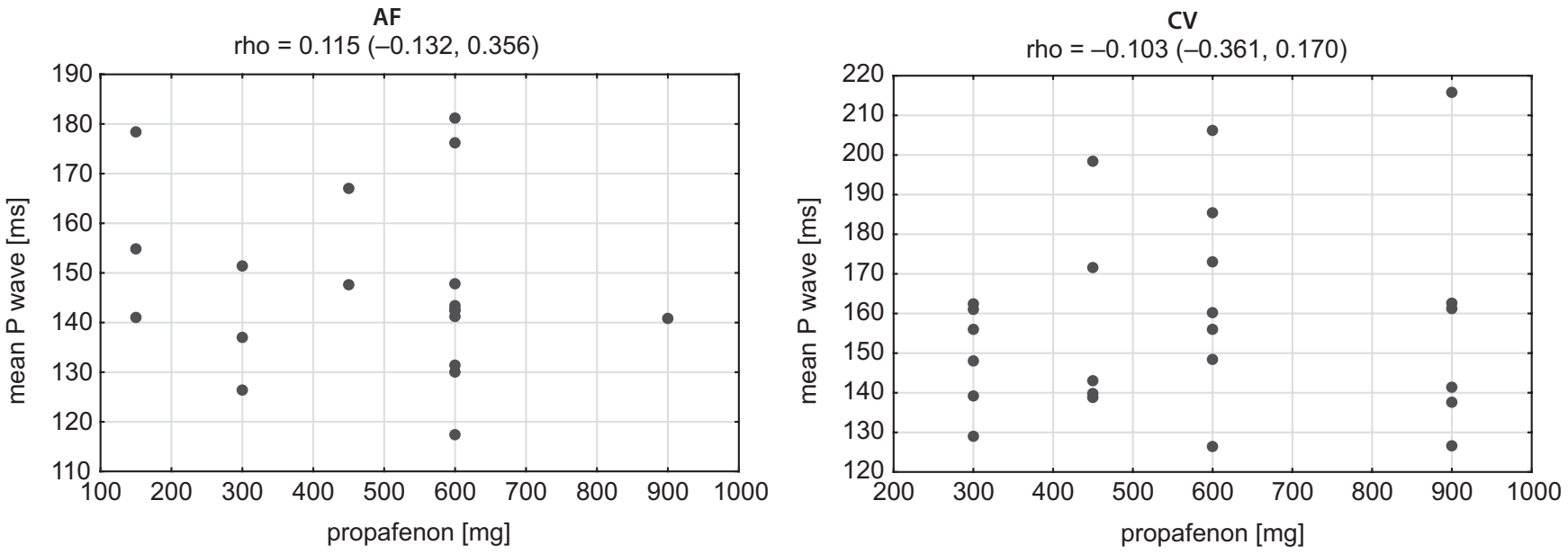

Fig. 4. Correlation diagram of average $\mathrm{P}$ wave duration with creatinine level and Spearman's rank correlation coefficient and its $95 \% \mathrm{Cl}$ 
Table 2 . Basic statistics of the average P wave duration in the study group according to the analyzed parameters and the results of significance tests

\begin{tabular}{|c|c|c|c|c|}
\hline \multirow{2}{*}{ Variables } & \multicolumn{3}{|c|}{ Mean $\mathrm{P}$ wave duration [ms] } & \multirow{2}{*}{$p$-value } \\
\hline & $\mathrm{M} \pm \mathrm{SD}$ & $\mathrm{Me}[\mathrm{Q} 1, \mathrm{Q} 3]$ & $\min -\max$ & \\
\hline \multicolumn{4}{|c|}{ Group } & \multirow{3}{*}{$<0.001$} \\
\hline $\operatorname{AF}(n=65)$ & $144.6 \pm 17.2$ & $141[133 ; 154]$ & $116-196$ & \\
\hline$C V(n=54)$ & $159.9 \pm 22.3$ & $159[139 ; 174]$ & $126-216$ & \\
\hline \multicolumn{4}{|c|}{ Gender } & \multirow{3}{*}{0.273} \\
\hline Male $(n=62)$ & $149.4 \pm 20.2$ & $147[134 ; 162]$ & $116-206$ & \\
\hline Female $(n=57)$ & $153.8 \pm 21.8$ & $145[139 ; 167]$ & $118-216$ & \\
\hline \multicolumn{4}{|c|}{ Age [years] } & \multirow{3}{*}{0.193} \\
\hline$\leq 65(n=52)$ & $149.3 \pm 21.7$ & $141[136 ; 161]$ & $117-216$ & \\
\hline$>65(n=67)$ & $153.3 \pm 20.5$ & $150[139 ; 167]$ & 116-199 & \\
\hline \multicolumn{4}{|c|}{ HT } & \multirow{3}{*}{0.008} \\
\hline Yes $(n=89)$ & $154.4 \pm 21.7$ & $150[139 ; 168]$ & $116-216$ & \\
\hline No $(n=30)$ & $142.9 \pm 16.2$ & $139[132 ; 151]$ & $117-199$ & \\
\hline \multicolumn{4}{|c|}{ DM } & \multirow{3}{*}{0.891} \\
\hline Yes $(n=25)$ & $151.8 \pm 21.9$ & $145[139 ; 172]$ & $116-190$ & \\
\hline No $(n=94)$ & $151.5 \pm 20.9$ & $147[138 ; 163]$ & $117-216$ & \\
\hline \multicolumn{4}{|c|}{ CKD } & \multirow{3}{*}{0.002} \\
\hline Yes $(n=9)$ & $170.6 \pm 13.5$ & $172[163 ; 182]$ & 150-185 & \\
\hline No $(n=110)$ & $150.0 \pm 20.8$ & $143[137 ; 162]$ & $116-216$ & \\
\hline \multicolumn{4}{|c|}{$\mathrm{IHD}$} & \multirow{3}{*}{0.397} \\
\hline Yes $(n=21)$ & $154.7 \pm 22.8$ & $151[139 ; 177]$ & $116-186$ & \\
\hline No $(n=98)$ & $150.9 \pm 20.7$ & $145[137 ; 162]$ & $117-216$ & \\
\hline \multicolumn{4}{|c|}{$\mathrm{HF}$} & \multirow{3}{*}{0.361} \\
\hline Yes $(n=11)$ & $156.8 \pm 24.7$ & $160[139 ; 182]$ & 116-192 & \\
\hline No $(n=108)$ & $151.0 \pm 20.7$ & $145[137 ; 163]$ & $117-216$ & \\
\hline \multicolumn{4}{|c|}{ Propafenone } & \multirow{3}{*}{0.519} \\
\hline Yes $(n=43)$ & $153.2 \pm 22.0$ & $148[139 ; 163]$ & $117-216$ & \\
\hline No $(n=76)$ & $150.6 \pm 20.5$ & $145[136 ; 164]$ & 116-199 & \\
\hline \multicolumn{4}{|c|}{ Amiodarone } & \multirow{3}{*}{0.796} \\
\hline Yes $(n=17)$ & $150.3 \pm 17.8$ & $150[141 ; 160]$ & 116-182 & \\
\hline No $(n=102)$ & $151.7 \pm 21.6$ & $144[137 ; 167]$ & $117-216$ & \\
\hline \multicolumn{4}{|c|}{ Metoprolol } & \multirow{3}{*}{0.974} \\
\hline Yes $(n=86)$ & $151.2 \pm 20.9$ & $148[138 ; 163]$ & $116-216$ & \\
\hline No $(n=33)$ & $152.3 \pm 21.7$ & $141[137 ; 170]$ & $123-198$ & \\
\hline \multicolumn{4}{|c|}{ Bisoprolol } & \multirow{3}{*}{0.453} \\
\hline Yes $(n=22)$ & $147.6 \pm 17.7$ & $144[139 ; 156]$ & 125-199 & \\
\hline No $(n=97)$ & $152.4 \pm 21.7$ & $148[138 ; 167]$ & $116-216$ & \\
\hline
\end{tabular}

HT - arterial hypertension; DM - diabetes mellitus; CKD - chronic kidney disease; IHD - ischemic heart disease; HF - heart failure.

of patients differing according to the analyzed parameters and the results of significance tests are presented in Table 2.

In ANOVA, it turned out that $\mathrm{P}$ wave duration has a statistically significant relationship with diagnosis (AF group $=0 ;$ CV group $=1$ ) as well as hypertension and CKD. Due to the possibility of a strong correlation between these parameters, a multifactorial (progressive, stepwise) regression analysis was performed. As a result, the following model was obtained:
Mean P wave duration $=120.3+15.8 \times \mathrm{CV}+20.2 \times \mathrm{CKD}$ $+9.0 \times \mathrm{HT} \pm 18.5$

The factors (stimulants) of longer P wave duration turned out to be membership in the CV group and the presence of CKD and hypertension. All structural parameters of the model are statistically significant $(\mathrm{p}<0.0001)$ : $\mathrm{F}(3,115)=12.6 ; \mathrm{p}<0.001$

The duration of the $\mathrm{P}$ wave can be regarded as a parameter by which patients can be classified into the CV or AF 
Cut-off: P wave duration $>148 \mathrm{~ms}$

specificity $=0.677$

sensitivity $=0.667$

$\mathrm{AUC}=0.700(0.609-0.781)$

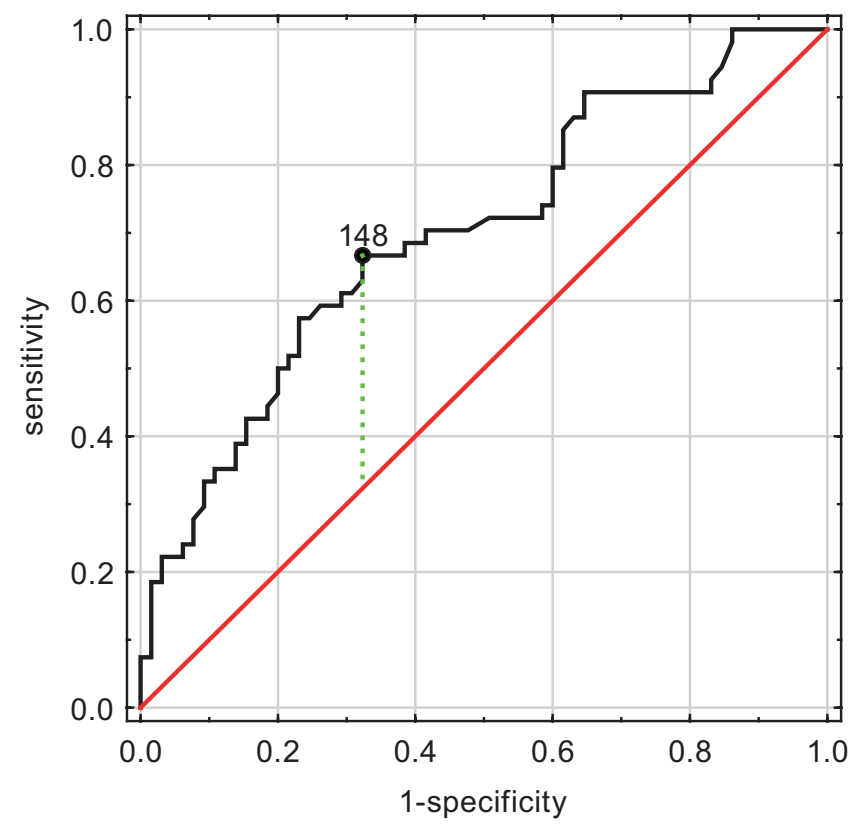

Fig. 5. ROC curve for $\mathrm{P}$ wave duration; cutoff value between patients from the $C V$ and the AF groups; the sensitivity and specificity of the test and the $\mathrm{AUC}$ and its $95 \% \mathrm{Cl}$

group. Analysis of the ROC curve showed that for the cutoff value of P wave, duration was lower than $148 \mathrm{~ms}$, the diagnostic sensitivity of the test was $67.7 \%$, the specificity $66.7 \%$, and the area under the ROC curve (AUC) was 0.700 . This is not a sensational result, but the lower confidence limit for AUC is 0.609 and is greater than 0.5, which indicates the diagnostic usefulness of this parameter. According to the literature, if the AUC is in the range of 0.7-0.8, the classifier is satisfactory. The graph is shown in Fig. 5.

\section{Discussion}

The duration of the $\mathrm{P}$ wave is a result of the conduction velocity in the atrial working myocardium and the distance the electrical activation has to travel. Functional and structural changes in the atrial muscle affect both of these parameters - they slow down conduction velocity and extend the path to be traveled due to the enlargement of the atria. This results in a longer $\mathrm{P}$ wave duration and a change in its morphology. The same changes in structure and function are responsible for generating atrial arrhythmias, including AF. ${ }^{6}$ Additionally, the ongoing arrhythmia leads to a progression of the abovementioned changes. In particular, the enlargement of the left atrium is clearly associated with an increase in the filling pressure of the left ventricle and likely dependent on the ventricular rate. ${ }^{10}$ Data on the effect of AF on muscle conduction is more scarce and less consistent. ${ }^{11}$
The main finding of our study is the longer duration of the $\mathrm{P}$ wave in patients with average long-term persistent AF compared to patients with paroxysmal arrhythmia and sinus rhythm at the time of the study. The degree to which the P wave duration was extended (about 10\%) is not only statistically significant, but clinically as well. It should be emphasized that the $\mathrm{P}$ waves measured in both groups markedly exceed the normal values of $120 \mathrm{~ms}(144.6 \mathrm{~ms}$ in AF group compared to $159.9 \mathrm{~ms}$ in CV group). Similar comparisons are not numerous in the available literature. ${ }^{12-13}$ As our subgroups of patients suffering from paroxysmal and persistent AF are comparable according to age and gender distribution, comorbidities and antiarrhythmic medication, it indicates that this additional prolongation was caused only by the presence of the prolonged episodes of arrhythmia. Our measurements were not performed immediately after the cardioversion shock, so the influence of direct current can be ignored. Additionally, in 1 small study, the direct current flow during cardioversion had little or no effect on the P wave duration immediately after the procedure and on the next day. ${ }^{14}$

Even if diabetic patients were not frequently presented in our study group, there was a noticeable difference between diabetics and non-diabetics in terms of $\mathrm{P}$ wave duration. Those with diabetes mellitus (DM) were observed to have a longer $\mathrm{P}$ wave than participants without it. This is in line with other clinical observations even the direct evidence lacks in human. Diabetes is presumed to be a risk factor for AF; the topic has been reviewed quite often. A meta-analysis of different cohort and case control studies investigating the correlation between DM and AF showed that individuals with DM had a $40 \%$ greater risk of AF than unaffected individuals. ${ }^{15}$ There is sparse literature to be found about DM leading to electrical changes of the atrial substrate. ${ }^{16}$ In an experimental setting of DM, it was associated with increased atrial fibrosis, interatrial conduction delay and greater inducibility of AF. ${ }^{17}$ Another animal study confirmed those results with additional interesting observations of $\mathrm{P}$ wave prolongation in diabetic rats without left atrial enlargement, which the authors attributed to diabetic changes in the gap junction protein, $\mathrm{Cx} .{ }^{18} \mathrm{Simi}$ lar outcomes were obtained in patients with impaired fasting glucose levels leading to significantly longer interatrial conduction times and, in consequence, decreased left atrial emptying volume and fraction. ${ }^{19}$

In another subgroup of our patients, CKD was found to be a predictor of longer $\mathrm{P}$ wave duration. In the literature, some studies have made an association between maximum $P$ wave duration and an exacerbation of the renal condition until the defined endpoints of hemodialysis, death or a specified decline in estimated glomerular filtration rate (eGFR). ${ }^{20,21}$ Based on our results, it could be assumed that a reciprocal influence of CKD on P wave duration is occurring as well, possibly because of a simple fluid overload. Referring to the patients included in our study, only a small number of patients $(9(7.6 \%))$ presented with 
CKD as a comorbidity, but it was discovered to be statistically significantly related to $\mathrm{P}$ wave duration. This requires further investigation in other studies which would not be distorted by the small number of CKD patients. Atrial fibrillation is frequently described together with renal dysfunction, mainly as a preceding comorbidity, but no evidence has been found supporting the notion of CKD being the cause of AF. Nevertheless, our results indicate such a possibility, making the subject worthy of study.

Anti-arrhythmic medication influences the electrophysiological properties of the working myocardium, in particular the conduction speed and refractory period, which could influence $\mathrm{P}$ wave duration. The results of our study do not support this idea. Amiodarone is a class III antiarrhythmic agent acting mainly as a potassium channel blocker. The drug prolongs refractory period and atrial repolarization. It has been shown to be effective in maintaining a sinus rhythm and preventing arrhythmia episodes in patients with paroxysmal AF. Although in 1 small study the researchers reported an amiodarone-related increase in $\mathrm{P}$ wave duration, it was a narrow, experimental animal study and the conditions were not comparable to the sinus rhythm in humans, which was present in our study. ${ }^{22}$ The relationship between $\mathrm{P}$ wave duration and amiodarone administration was similarly negated in a study conducted by Sasaki et al. ${ }^{23}$

In contrast to amiodarone, treatment with a sodium channel blocker could theoretically influence the P wave duration. Propafenone is an IC class agent which blocks the fast sodium channels, slowing down the conduction velocity in the working myocardium. According to literature data, there is no direct relationship between the dosage of propafenone and $\mathrm{P}$ wave duration, though the same study confirmed a weak correlation between treatment with propafenone and a prolongation of $\mathrm{P}$ wave duration. ${ }^{24}$ Our data does not confirm this finding. It should be noted, however, that our group of patients treated with propafenone was not very large.

Based on our results, the theoretical model resulting from ROC curves indicates an estimated $\mathrm{P}$ wave duration which differentiates patients between sinus rhythm and persistent AF groups. This approach has not been presented in the literature so far, so our value of $P$ wave duration $(148 \mathrm{~ms})$ can only be referred to studies indicating the importance of this parameter in the prediction of sinus rhythm maintenance after electrical cardioversion. In 1999, Aytemir et al. investigated the P wave signal-averaged ECG in 73 patients after successful cardioversion. During the six-month follow-up period, a recurrence of AF was observed in 31 patients, while in 42 patients, a sinus rhythm was maintained. The researchers found no difference between the groups according to gender, age, presence of organic heart disease, left atrial diameter, left ventricular ejection fraction, use of antiarrhythmic drug, or duration of AF. The filtered P wave duration was statistically significantly longer in patients with a recurrence of AF
(138.4 ms compared to $112.5 \mathrm{~ms}$ ). A filtered P wave duration of $128 \mathrm{~ms}$ had a sensitivity of $70 \%$ and a specificity of $76 \%$ for the detection of AF recurrence. ${ }^{25}$ On the other hand, in a study by Perzanowski et al. the maximum P wave duration did not differentiate patients who remained in sinus rhythm or experienced a recurrence of arrhythmia (142 ms compared to $145 \mathrm{~ms}$; p-value not significant). ${ }^{26}$ As the authors did not mention their methodology of P wave duration measurement, it should be assumed that they used a simple, standard 12-lead ECG without any more precise equipment. This lack of precision could have been the cause of their results. In a study by Gonna et al., a 12-lead ECG was recorded after electrical cardioversion for persistent AF in 77 patients and was repeated after 1 month. Compared with the sinus rhythm group, the group with recurrent $A F$ had more patients with a $P$ wave duration that exceeded $142 \mathrm{~ms}$. Using a cutoff $<142 \mathrm{~ms}$ for P wave duration, the authors showed a sensitivity of $64.6 \%$ and a specificity of $62.1 \%$ for sinus rhythm maintenance. In multiple regression analysis, a P wave duration longer than $142 \mathrm{~ms}$ was the only independent predictor of AF recurrence. ${ }^{27}$ The abovementioned considerations indicate unequivocally that the prolongation of the $\mathrm{P}$ wave is clearly a risk factor for paroxysm of AF and for more advanced stages of arrhythmia, which is in line in our results. Moreover, we have produced evidence which supports the previous findings in different settings. Regarding the higher values of $\mathrm{P}$ wave duration obtained in this study, it should be remembered that the precise methodology used in our study is qualitatively different from that of other researchers. ${ }^{26,27}$ This is because a few years ago we confirmed the lack of $\mathrm{P}$ wave dispersion that was reported in some of the abovementioned papers, which is related to the inaccuracy of the measurements. ${ }^{28}$

In summary, ongoing AF in the form of a moderately long, persistent arrhythmia has a negative impact on structural and functional atrial remodeling. This occurs independently of age, gender and type of anti-arrhythmic treatment, it but may be somewhat related to certain comorbidities.

\section{Study limitations}

An important limitation of our study is its single-center design and relatively small study group. In addition, this is not a prospective clinical study indicating the relationship between ECG parameters and long-term prognosis. As our measurement method - an electrophysiological recording system - is extremely precise, we are aware that our results cannot be directly compared to those of other authors. We previously mentioned that the duration of the persistent form of arrhythmia cannot be exactly estimated. Because our hypothesis is that the longer P wave duration is mainly related to AF itself, this should be assumed as the main limitation of our study, even if the literature data contradict the correlation between arrhythmia duration and $\mathrm{P}$ wave prolongation. ${ }^{14}$ 


\section{Conclusions}

Persistent AF shows a longer $\mathrm{P}$ wave duration than the paroxysmal form of arrhythmia, independent of age, gender and anti-arrhythmic medication. The prolongation of the P wave related to persistent arrhythmia should compel physicians to restore a sinus rhythm earlier to more successfully maintain it in the long term.

\section{ORCID iDs}

Malte Unkell (10 https://orcid.org/0000-0003-4471-848X Maxim Marinov (1) https://orcid.org/0000-0001-8048-9675 Peter Stephan Wolff (10 https://orcid.org/0000-0002-9687-8808 Jadwiga Radziejewska (1) https://orcid.org/0000-0001-9153-9754 Jakub Szymon Mercik (1) https://orcid.org/0000-0002-5627-7071 Jacek Gajek (1) https://orcid.org/0000-0002-0038-1750

\section{References}

1. Thijssen VLJL, Ausma J, Liu GS, Allessie MA, van Eys GJ, Borgers M. Structural changes of atrial myocardium during chronic atrial fibrillation. Cardiovasc Pathol. 2000;9(1):17-28.

2. Casaclang-Verzosa G, Gersh BJ, Tsang TSM. Structural and functional remodeling of the left atrium: Clinical and therapeutic implications for atrial fibrillation. J Am Coll Cardiol. 2008;51(1):1-11.

3. Waks JW, Josephson ME. Mechanisms of atrial fibrillation: Reentry, rotors and reality. Arrhythm Electrophysiol Rev. 2014;3(2):90-100.

4. Pellman J, Sheikh F. Atrial fibrillation: Mechanisms, therapeutics and future directions. Compr Physiol. 2015;5(2):649-665.

5. Rosso R, Kistler PM. Focal atrial tachycardia. Heart. 2010;96(3):181-185.

6. Hari KJ, Nguyen TP, Soliman EZ. Relationship between P-wave duration and the risk of atrial fibrillation. Expert Rev Cardiovasc Ther. 2018; 16(11):837-843.

7. Wijffels $M C$, Kirchhof CJ, Dorland R, Allessie MA. Atrial fibrillation begets atrial fibrillation: A study in awake chronically instrumented goats. Circulation. 1995;92(7):1954-1968.

8. Shukla A, Curtis AB. Avoiding permanent atrial fibrillation: Treatment approaches to prevent disease progression. Vasc Health Risk Manag. 2014;10(1):1-12.

9. Fuster V, Rydén LE, Cannom DS, et al; American College of Cardiology Foundation/American Heart Association Task Force. 2011 ACCF/ AHA/HRS focused updates incorporated into the ACC/AHA/ESC 2006 guidelines for the management of patients with atrial fibrillation: A report of the American College of Cardiology Foundation/American Heart Association Task Force on practice guidelines. Circulation. 2011;123(10):e269-e367.

10. D'Andrea A, De Corato G, Scarafile R, et al. Left atrial myocardial function in either physiological or pathological left ventricular hypertrophy: A two-dimensional speckle strain study. Br J Sports Med. 2008; 42(8):696-702.
11. Nattel S, Burstein B, Dobrev D. Atrial remodeling and atrial fibrillation: Mechanisms and implications. Circ Arrhythm Electrophysiol. 2008;1(1):62-73.

12. Censi F, Calcagnini G, Triventi M, et al. P-wave characteristics after electrical external cardioversion: Predictive indexes of relapse. Conf Proc IEEE Eng Med Biol Soc. 2010:3442-3445.

13. Bagliani G, Michelucci A, Angeli F, Meniconi L. Atrial activation analysis by surface $P$ wave and multipolar esophageal recording after cardioversion of persistent atrial fibrillation. Pacing Clin Electrophysiol. 2003;26(5):1178-1188.

14. Sato T, Mitamura H, Kurita $\mathrm{Y}$, et al. Recovery of electrophysiological parameters after conversion of atrial fibrillation. Int J Cardiol. 2001; 79(2-3):183-189.

15. Huxley RR, Filion KB, Konety S, Alonso A. Meta-analysis of cohort and case-control studies of type 2 diabetes mellitus and risk of atrial fibrillation. Am J Cardiol. 2011;108(1):56-62.

16. Wang A, Green JB, Halperin, Piccini JP Sr. Atrial fibrillation and diabetes mellitus. J Am Coll Cardiol. 2019;74(8):1107-1115.

17. Fu H, Liu C, Li J, et al. Impaired atrial electromechanical function and atrial fibrillation promotion in alloxan-induced diabetic rabbits. Cardiol J. 2013;20(1):59-67.

18. Li B, Pan Y, Li X. Type 2 diabetes induces prolonged P-wave duration without left atrial enlargement. J Korean Med Sci. 2016;31(4):525-534.

19. Ayhan S, Ozturk S, Alcelik A, et al. Atrial conduction time and atrial mechanical function in patients with impaired fasting glucose. J Interv Card Electrophysiol. 2012;35(3):247-252.

20. Huang J-C, Wei S-Y, Chen S-C, et al. P wave dispersion and maximum $P$ wave duration are associated with renal outcomes in chronic kidney disease. PLoS One. 2014;9(7):e101962.

21. Su H-M, Tsai W-C, Lin T-H, et al. P wave dispersion and maximum $P$ wave duration are independently associated with rapid renal function decline. PLoS One. 2012;7(8):e42815.

22. Burashnikov A, Di Diego JM, Sicouri S, Ferreiro M, Carlsson L, Antzelevitch $C$. Atrial-selective effects of chronic amiodarone in the management of atrial fibrillation. Heart Rhythm. 2008;5(12):1735-1742.

23. Sasaki N, Watanabe I, Kogawa R, et al. Effects of intravenous amiodarone and ibutilide on action potential duration and atrial conduction kinetics in patients with persistent atrial fibrillation. Int Heart J. 2014;55(3):244-248.

24. Cheriex EC, Krijne R, Brugada P, Heymeriks J, Wellens HJ. Lack of clinically significant beta-blocking effect of propafenone. Eur Heart J. 1986;8(1):53-56.

25. Aytemir K, Aksoyek S, Yildirir A, Ozer N, Oto A. Prediction of atrial fibrillation recurrence after cardioversion by $P$ wave signal-averaged electrocardiography. Int J Cardiol. 1999;70(1):15-21.

26. Perzanowski C, Ho AT, Jacobson AK. Increased P-wave dispersion predicts recurrent atrial fibrillation after cardioversion. J Electrocardiol. 2005;38(1):43-46.

27. Gonna H, Gallagher MM, Guo XH, Yap YG, Hnatkova J, Camm AJ. $\mathrm{P}$-wave abnormality predicts recurrence of atrial fibrillation after electrical cardioversion: A prospective study. Ann Noninvasive Electrocardiol. 2014;19(1):57-62.

28. Zimmer K, Przywara W, Zyśko D, Sławuta A, Gajek J. The nature of P-wave dispersion: A clinically useful parameter that does not exist. Int J Cardiol. 2016;212:59-60. 